\title{
VECTOR BUNDLES AND PROJECTIVE MODULES
}

\author{
BY
}

LEONID N. VASERSTEIN ${ }^{1}$

\begin{abstract}
Serre and Swan showed that the category of vector bundles over a compact space $X$ is equivalent to the category of finitely generated projective modules over the ring of continuous functions on $X$. In this paper, titled after the famous paper by Swan, this result is extended to an arbitrary topological space $X$. Also the well-known homotopy classification of the vector bundles over compact $X$ up to isomorphism is extended to arbitrary $X$. It is shown that the $K_{0}$-functor and the Witt group of the ring of continuous functions on $X$ coincide, and they are homotopy-type invariants of $X$.
\end{abstract}

1. Statement of results. Let $F$ be either the real numbers $R$, the complex numbers $C$, or the quaternions $H$. For a topological space $X$, let $F^{X}$ denote the ring of all continuous functions $X \rightarrow F$.

When $X$ is compact Hausdorff, it is well known (Swan [5]) that the category $P\left(F^{X}\right)$ of finitely generated projective $F^{X}$-modules is equivalent to the category of $F$-vector bundles over $X$. Later Goodearl $[\mathbf{2}]$ observed that the equivalence holds in the more general case of paracompact Hausdorff $X$ if we restrict ourselves to the bundles of finite type. This restriction excludes vector bundles of unbounded dimension which cannot come from $P\left(F^{X}\right)$.

The first goal of this paper is to extend this result (with an appropriate definition of finite type) to an arbitrary topological space $X$.

DEFINITION. A bundle over $X$ (see [5]) is of finite type if there is a finite partition $S$ of 1 on $X$ (i.e. a finite set $S$ of nonnegative continuous functions on $X$ whose sum is 1) such that the restriction of the bundle to the set $\{x \in X: f(x) \neq 0\}$ is trivial for each $f$ in $S$.

For example, when $X$ is compact Hausdorff, every bundle over $X$ is of finite type. For a normal space $X$ our definition of finite type is equivalent to the following definition [2]: there is a finite open covering $T$ of $X$ such that the restriction of the bundle to each $U \in T$ is trivial.

THEOREM 1. The category $\mathcal{P}\left(F^{X}\right)$ is equivalent to the category of $F$-vector bundles of finite type over $X$.

Our second theorem concerns the classification problem for the bundles of finite type over a topological space $X$, i.e. the problem of identification of the isomorphism classes in $\mathcal{P}\left(F^{X}\right)$ with homotopy classes. Let $G\left(F^{n}\right)$ be the set of all subspaces of the $n$-dimensional vector space $F^{n}$ over $F$. As usual, $G\left(F^{n}\right)$ is endowed with

Received by the editors May 18, 1985. Some results of this paper were reported by the author on March 20, 1985 in Oberwolfach, West Germany, under the title Quadratic forms over rings of continuous functions at the meeting on "Surgery and $L$-theory".

1980 Mathematics Subject Classification. Primary 18F25.

Key words and phrases. Projective modules, vector bundles, Witt group.

${ }^{1}$ The author is a John Simon Guggenheim fellow and supported in part by the NSF. 
the topology of the disjoint union of the Grassmann manifolds $G_{m}\left(F^{n}\right)$, where $0 \leq m \leq n$. The topology on $G\left(F^{n}\right)$ can be given explicitly by identifying $G\left(F^{n}\right)$ with the subset $Y_{1}=\left\{p=p^{2}=p^{*} \in M_{n} F\right\}$ or $Y_{2}=\left\{a \in M_{n} F: a^{2}=a a^{*}=1_{n}\right\}$ of the matrix ring $M_{n} F$ (which has the topology of the direct product of $n^{2}$ copies of $F$ ). The following 2 subsets of $M_{n} F$ are homotopically equivalent to $G\left(F^{n}\right)$, so they can replace $G\left(F^{n}\right)$ in Theorem 2 below: $Y_{3}=\left\{e=e^{2} \in M_{n} F\right\}, Y_{4}=\{a=$ $\left.a^{*} \in \mathrm{GL}_{n} F\right\}$. The inclusion $F^{n} \subset F^{n+1}$ induces the inclusion $G\left(F^{n}\right) \subset G\left(F^{n+1}\right)$ which corresponds to the map $b \mapsto\left(\begin{array}{ll}b & 0 \\ 0 & 0\end{array}\right)$ on the sets $Y_{1}$ and $Y_{3}$ and to the map $b \mapsto\left(\begin{array}{cc}b & 0 \\ 0 & -1\end{array}\right)$ on the sets $Y_{2}$ and $Y_{4}$.

THEOREM 2. There is a bijection between any two of the following five sets:

(1) the isomorphism classes of $F$-vector bundles of finite type over $X$;

(2) the isomorphism classes in $P\left(F^{X}\right)$;

(3) the isomorphism classes in $P\left(F_{0}^{X}\right)$, where $F_{0}^{X} \subset F^{X}$ is the subring of bounded functions on $X$;

(4) the isomorphism classes of finitely generated projective $F^{X}$-modules with positive definite hermitian forms;

(5) the inductive limit of the homotopy classes of continuous maps from $X$ to $G\left(F^{n}\right)$ as $n \rightarrow \infty$.

COROLlaRY. The set of isomorphism classes in $\mathcal{P}\left(F^{X}\right)$ is a homotopy-type invariant of $X$. In particular, $K_{0} F^{X}$, the Grothendieck group of $\mathcal{P}\left(F^{X}\right)$, is a homotopy-type invariant of $X$.

REMARK. The rings $F^{X}$ and $F_{0}^{X}$ in Theorem 2 can be replaced by dense subrings (see [6]). For example, if $X$ is a manifold and $A$ is the ring of smooth functions on $X$, then there is a bijection between the isomorphism classes in $P\left(F^{X}\right)$ and $P(A)$. In particular, $K_{0} F^{X}=K_{0} A$ is a homotopy-type invariant of $X$. Note that the algebraic $K_{0}$-functor does not take into account any topology on rings.

Our third goal is to compute the Witt group of hermitian forms over $F^{X}$. Let $P^{+}\left(F^{X}\right)$ denote the category of hermitian spaces $(P, \Phi)$ over $F^{X}$, where $P \in \mathcal{P}\left(F^{X}\right)$ and $\Phi$ is a nonsingular hermitian form on $P$ (nonsingular here means that the corresponding map $P \rightarrow P^{*}$ is an isomorphism). The morphisms in $P^{+}\left(F^{X}\right)$ preserve scalar products. In particular, they have trivial kernels.

THEOREM 3. The set of isomorphism classes in $\mathrm{P}^{+}\left(F^{X}\right)$ is the direct square of the set of isomorphism classes of objects in $P\left(F^{X}\right)$.

COROLlarY. The Grothendieck group of the category $\mathrm{P}^{+}\left(F^{X}\right)$, i.e. the WittGrothendieck group of $F^{X}$, is isomorphic to $K_{0} F^{X} \times K_{0} F^{X}$. The Witt group of $F^{X}$ is isomorphic to $K_{0} F^{X}$.

Recall that the Witt group is the cokernel of the homomorphism of the Grothendieck groups induced by the hyperbolic functor $\mathcal{P}\left(F^{X}\right) \rightarrow \mathcal{P}^{+}\left(F^{X}\right)$.

\section{Proof of Theorem 1.}

LEMMA 4. Every object $P$ in $P\left(F^{X}\right)$ is isomorphic to the column space of a square hermitian idempotent matrix $e=e^{2}=e^{*}$ over $F^{X}$.

PROOF. Since $P$ is isomorphic to a direct summand of the free module $\left(F^{X}\right)^{n}$ for some natural number $n$, it is isomorphic to the column space (i.e. to the image 
$\left.a\left(F^{X}\right)^{n}\right)$ of an $n$-by-n idempotent matrix $a=a^{2}$ over $F^{X}$. Since $a=a^{2}$, we have $\left(a^{*}\right)^{2}=a^{*}$ and

$$
\left(1+(1-2 a)\left(1-2 a^{*}\right)\right) a^{*}=2 a a^{*}=a\left(1+(1-2 a)\left(1-2 a^{*}\right)\right),
$$

where 1 stands for the identity matrix $1_{n} \in M_{n} F^{X}$. Since $(1-2 a)\left(1-2 a^{*}\right)$ is a hermitian semipositive matrix, there is a hermitian matrix $g=g^{*}$ in $\mathrm{GL}_{n} F^{X}$ such that $g^{2}=1+(1-2 a)\left(1-2 a^{*}\right)$. We set $e=g a g^{-1}$. Then $e^{2}=g a^{2} g^{-1}=g a g^{-1}=e$ and $e^{*}=g^{-1} a^{*} g=g a g^{-1}=e$. So $P$ is isomorphic to the column space of this $e=e^{2}=e^{*}$. The lemma is proved.

Every object $P$ in $P\left(F^{X}\right)$ gives an $F$-vector bundle $\Gamma^{*}(P)$ over $X$ in the usual way. Namely, the $F$-vector space at a point $x$ of $X$ is $P$ evaluated at $x$; i.e. $P \otimes_{v} F^{X}$, where $v: F^{X} \rightarrow F$ is given by $v(f)=f(x)$ for any continuous function $f: X \rightarrow F$. When $P$ is the column space of a matrix $e=e^{2} \in M_{n} F^{X}$, the fiber at $x$ is just $e(x) F^{n}$. The local triviality of $\Gamma^{*}(P)$ follows from the following lemma.

LEMMA 5. If $e=e^{2} \in M_{n} F^{X}, x, y \in X$, and $g=e(x) e(y)+(1-e(x))(1-e(y)) \in$ $\mathrm{GL}_{n} F$, then the matrices $e(x)$ and $e(y)$ over $F$ are similar.

ProOF. We have $e(x) g=g e(y)$. When $g \in \mathrm{GL}_{n} F$, we conclude that $g^{-1} e(x) g=$ $e(y)$. The lemma is proved.

COROLlaRY 6. For every object $P$ in $P\left(F^{X}\right)$, the corresponding $F$-vector bundle $\Gamma^{*}(P)$ over $X$ is of finite type.

Proof. By Lemma 4, $P$ is isomorphic to the column space of $e=e^{2}=e^{*} \in$ $M_{n} F^{X}$ for some $n, e$. The set $Y_{1}=\left\{p=p^{2}=p^{*} \in M_{n} F\right\}$ is compact (note that $|p| \leq 1$ for each $p$ in $Y_{1}$ ). Therefore there is a finite partition $S^{\prime}$ of 1 on $Y_{1}$ such that $|p-q|<1 / 3$ whenever $f^{\prime}(p) f^{\prime}(q) \neq 0$ for some $f^{\prime}$ in $S^{\prime}$. (Note that $F^{n}$ has the usual metric $\left|\left(z_{j}\right)\right|^{2}=\sum z_{j}^{*} z_{j}$ which makes it a Banach space, so $M_{n} F$ is a Banach algebra with respect to the operator norm.)

The matrix $e$ above can be considered as a continuous map $X \rightarrow Y_{1}$. So the partition $S^{\prime}$ gives a finite partition $S$ of 1 on $X$. We have $|e(x)-e(y)|<1 / 3$ whenever $x$ and $y$ are in the same part (i.e. $f(x) f(y) \neq 0$ for some $f$ in $S$ ).

For any part $U(f)=\{x \in X: f(x) \neq 0\}$, where $f \in S$, and any $x, y$ in $U(f)$ we have, as in Lemma $5, e(x) g=g e(y)$, where

$$
g=e(x) e(y)+(1-e(x))(1-e(y))=1-(e(y)-e(x))(1-2 e(y)) .
$$

Since

$$
|g-1|=|(e(y)-e(x))(1-2 e(y))| \leq|e(y)-e(x)||1-2 e(y)|<(1 / 3) \cdot 3=1,
$$

we conclude that $g \in \mathrm{GL}_{n} F$. Fixing $x \in U(f)$, we have $e(y)=g^{-1} e(x) g$, where $g$ depends continuously on $y \in U(f)$. Since $F$ is a division algebra, the column space of $e(z)$ is a finite dimensional vector space over $F$ for each $z$ in $X$. Therefore the column space of the restriction of $e$ to $U(f)$ is a finitely generated free $F^{U(f)}$ module. So the restriction of the bundle $\Gamma^{*}(P)$ to $U(f)$ is trivial. Thus, the bundle is of finite type. The corollary is proved.

LEMMA 7. For every $F$-vector bundle $\xi$ of finite type over $X$, the $F^{X}$-module $\Gamma(\xi)$ of its global sections is finitely generated and projective (i.e. $\Gamma(\xi) \in P\left(F^{X}\right)$ ).

PROOF. Let $S$ be a finite partition of 1 on $X$ such that $\xi$ is trivial over each $U(f)=\{x \in X: f(x) \neq 0\}, f \in S$. For each $f$ in $S$ we pick a free basis 
$a_{1}(f), \ldots, a_{n(f)}(f)$ for $\left.\Gamma(\xi)\right|_{U(f)}$. We would like to extend these $a_{j}(f)$ to global sections of the bundle. However, in general, this can be done only after a modification of $a_{j}(f)$.

We write $a_{j}(f)=\sum c_{i, j}(f, g) a_{i}(g)$ on $U(f) \cap U(g)$ for every $g$ in $S$. It is clear that there is a continuous nonnegative function $f^{\prime}$ on $X$ such that $f^{\prime}$ and $f$ have the same zero set and $c_{i, j}(f, g) f^{\prime} \rightarrow 0$ on $U(f) \cap U(g)$ whenever $f(x) \rightarrow 0$, for all $i, j$ and $g$. Then

$$
b_{j}(f)= \begin{cases}a_{j}(f) f^{\prime} & \text { on } U(f), \\ 0 & \text { elsewhere on } X\end{cases}
$$

is a continuous global section of the bundle. Moreover, $\left\{\left.b_{j}(f)\right|_{U(f)}: 1 \leq j \leq n(f)\right\}$ is a basis for $\left.\Gamma(\xi)\right|_{U(f)}$.

Let us show now that $\left\{b_{j}(f): 1 \leq j \leq n(f), f \in S\right\}$ is a generating set for the $F^{X}$. module $\Gamma(\xi)$. Let $s \in \Gamma(\xi)$. We write $\left.s\right|_{U(f)}=\sum c_{j}(f) b_{j}(f)$ with $c_{j}(f) \in F^{U(f)}$. There is a modification (depending on $s$ ) of the partition $S$ such that the open covering $\{U(f): f \in S\}$ of $X$ stays the same, but for the new partition we have that $c_{i}(f) f \rightarrow 0$ on $U(f)$ whenever $f \rightarrow 0$. Then $d_{j}(f) \in F^{X}$ for $d_{j}(f)$ defined by

$$
d_{j}(f)= \begin{cases}c_{j}(f) f & \text { on } U(f) \\ 0 & \text { elsewhere on } X .\end{cases}
$$

Moreover,

$$
s=\sum_{f \in S} \sum_{s=1}^{n(f)} d_{j}(f) b_{j}(f) .
$$

Therefore we have a surjective map from $\left(F^{X}\right)^{N}$, where $N=\sum_{f \in S} n(f)$ is the number of the generators, onto $\Gamma(\xi)$. Using a positive definite hermitian form on $\left(F^{X}\right)^{N}$ (say, the standard form $(a, b) \mapsto \sum a_{j}^{*} b_{j}$ for $a=\left(a_{j}\right), b=\left(b_{j}\right)$ in $\left(F^{X}\right)^{N}$ ), we obtain, as in [5], that the kernel of the homomorphism $\left(F^{X}\right)^{N} \rightarrow \Gamma(\xi)$ is a direct summand of $\left(F^{X}\right)^{N}$. So $\Gamma(\xi)$ is a projective $F^{X}$-module. Thus, Lemma 7 is proved.

Putting together Lemmas 4 and 7, we conclude, as in [5] (see also [3 and 4]) that the functors $\Gamma$ and $\Gamma^{*}$ give an equivalence of the category $P\left(F^{X}\right)$ and the category of $F$-vector bundles over $X$ of finite type.

\section{Proof of Theorem 2.}

Isomorphism of the sets (1) and (2). This follows from Theorem 1.

Isomorphism of the sets (2) and (3). By Lemma 4 every object $P$ of $P\left(F^{X}\right)$ is isomorphic to an object extended from some object of $P\left(F_{0}^{X}\right)$. Let now $P$ and $Q$ be objects of $P\left(F_{0}^{X}\right)$ which are isomorphic over the bigger $\operatorname{ring} F^{X}$. We want to prove that $P$ and $Q$ are isomorphic in $P\left(F_{0}^{X}\right)$.

We represent $P$ and $Q$ as the column spaces of matrices $p=p^{2}=p^{*}$ and $q=q^{2}=q^{*}$ in $M_{n} F_{0}^{X}$. Using the stabilization operations $e \mapsto\left(\begin{array}{ll}e & 0 \\ 0 & 0\end{array}\right)$ (which allowed us to take the same $n$ for $p$ and $q$ ), and an isomorphism of $P$ and $Q$ over $F^{X}$, we can assume that $g^{-1} p g=q$ for some $g \in \mathrm{GL}_{n} F^{X}$.

Let $g=g^{\prime} h$ be the polar decomposition of $g$, i.e. $g^{\prime}=g^{\prime *}$ is hermitian, $h=h^{*-1}$ is orthogonal (unitary), $g^{\prime 2}=g g^{*}$, and the centralizer of $g^{\prime}$ in $M_{n} F^{X}$ is the same as that of $g g^{*}$, so $p g^{\prime}=g^{\prime} p$. Then $h \in \mathrm{GL}_{n} F_{0}^{X}$ is bounded and $h^{-1} p h=h^{*} p g=q$. So $P$ and $Q$ are isomorphic over $F_{0}^{X}$. 
Isomorphism of the sets (2) and (4). Let us show that every object $P$ in $P\left(F^{X}\right)$ carries a positive definite hermitian form. By Lemma $4, P$ is isomorphic to the column space $e\left(F^{X}\right)^{n}$ of a matrix $e=e^{2}=e^{*}$ in $M_{n} F^{X}$. We define a hermitian positive definite form

$$
\Phi: e\left(F^{X}\right)^{n} \times e\left(F^{X}\right)^{n} \rightarrow F^{X}
$$

by

$$
\Phi\left(\left(a_{i}\right),\left(b_{i}\right)\right)=\sum a_{i}^{*} b_{i} .
$$

Note that $(1-e)\left(F^{X}\right)^{n}$ is the orthogonal complement to $e\left(F^{X}\right)^{n}$.

Let us show now that any two positive definite hermitian forms $\Phi$ and $\Psi$ on $P$ are isomorphic. Following [4, Theorem 8.8], consider $h=\Phi^{-1} \Psi \in \operatorname{Aut}(P)$ (where $\Phi$ and $\Psi$ are regarded as maps $\left.P \rightarrow P^{*}\right)$. Then $h$ is selfadjoint and positive with respect to $\Phi$ (namely,

$$
\begin{aligned}
\Phi(h u, v) & =(\Phi h u) v=(\Psi u) v=\Psi(u, v)=\Psi(v, u)^{*} \\
& =(\Phi(h v, u))^{*}=\Phi(u, h v)^{* *}=\Phi(u, h v)
\end{aligned}
$$

and $\Phi(h v, v)=\Psi(v, v)>0$ for $0 \neq v \in P)$. Let $g$ be its selfadjoint positive square root. Then $\Psi=\Phi h=\Phi g^{2}=g^{*} \Phi g$ (i.e. $\Psi(u, v)=\Phi(h u, v)=\Phi\left(g^{2} u, v\right)=$ $\Phi(g u, g v))$, so $\Phi$ and $\Psi$ are isomorphic.

Isomorphism of the sets (2) and (5). We identify $G\left(F^{n}\right)$ with $\left\{p=p^{2}=p^{*} \in\right.$ $\left.M_{n} F\right\}$ and $\operatorname{Hom}\left(X, G\left(F^{n}\right)\right)$ (i.e. the continuous maps $\left.X \rightarrow G\left(F^{n}\right)\right)$ with $\left\{e=e^{2}=\right.$ $\left.e^{*} \in M_{n} F^{X}\right\}$.

By Lemma 4 , every object $P$ in $P\left(F^{X}\right)$ is isomorphic to the column space of such a matrix $e$. Let us show that the image of $e$ in $\lim \pi\left(X, G\left(F^{n}\right)\right)$ does not depend on the choice of $e$ (here $\pi\left(X, G\left(F^{n}\right)\right.$ ) denotes the homotopy classes in $\operatorname{Hom}\left(X, G\left(F^{n}\right)\right)$ ).

Let $a=a^{2}=a^{*}$ in $M_{m} F^{X}$ and $b=b^{2}=b^{*}$ in $M_{n} F^{X}$ have isomorphic column spaces. Using the stabilization maps $e \mapsto\left(\begin{array}{ll}e & 0 \\ 0 & 0\end{array}\right)$ which do not change images in the inductive limit, we can assume that $m=n$ and the matrices $a$ and $b$ are similar. That is, $b=g a g^{-1}$ with $g$ in $\mathrm{GL}_{n} F^{X}$.

Then we have, in $M_{2 n} F^{X}$, that

$$
\left(\begin{array}{cc}
g^{-1} & 0 \\
0 & g
\end{array}\right)\left(\begin{array}{ll}
a & 0 \\
0 & 0
\end{array}\right)\left(\begin{array}{cc}
g & 0 \\
0 & g^{-1}
\end{array}\right)=\left(\begin{array}{ll}
b & 0 \\
0 & 0
\end{array}\right) .
$$

By the Whitehead lemma, the matrix $\left(\begin{array}{cc}g & 0 \\ 0 & g^{-1}\end{array}\right)$ is the product of elementary matrices and, hence, homotopic to the identity matrix. So $\left(\begin{array}{ll}a & 0 \\ 0 & 0\end{array}\right)$ and $\left(\begin{array}{ll}b & 0 \\ 0 & 0\end{array}\right)$ are homotopic in the set $\left\{e=e^{2} \in M_{2 n} F^{X}\right\}$. This homotopy can be retracted to a homotopy in the subset $\left\{e=e^{2}=e^{*} \in M_{2 n} F^{X}\right\}$ (using, for example, the proof of Lemma 5 with $X$ replaced by $X \times\{t: 0 \leq t \leq 1\})$. Thus, $\left(\begin{array}{ll}a & 0 \\ 0 & 0\end{array}\right)$ and $\left(\begin{array}{ll}b & 0 \\ 0 & 0\end{array}\right)$ are homotopic in $\operatorname{Hom}\left(X, G\left(F^{2 n}\right)\right)$.

So we have a well-defined map from the isomorphism classes of objects in $P\left(F^{X}\right)$ to $\lim \pi\left(X, G\left(F^{n}\right)\right)$. This map is onto, as one can see by assigning to each $e=e^{2}=$ $e^{*}$ in $M_{n} F^{X}$ its column space, which gives a map from $\underset{\lim }{\longrightarrow}\left(\operatorname{Hom}\left(X, G\left(F^{n}\right)\right)\right)$ to $P\left(F^{X}\right)$ (up to isomorphism in $P\left(F^{X}\right)$, this map is onto by Lemma 4).

The following lemma completes our proof. 
LEMMA 8. Any two matrices in $\operatorname{Hom}\left(X, G\left(F^{n}\right)\right)$ which are homotopic are similar. In particular, their column spaces are isomorphic.

Proof. Let $a, b \in \operatorname{Hom}\left(X, G\left(F^{n}\right)\right)$ be homotopic. That is, there is a continuous map $c: X \times\{t: 0 \leq t \leq 1\} \rightarrow G\left(F^{n}\right)$ such that $c(\cdot, 0)=a$ and $c(\cdot, 1)=b$.

Let $\Pi G\left(F^{n}\right)$ denote the set of continuous maps $\{t: 0 \leq t \leq 1\} \rightarrow G\left(F^{n}\right)$. Then $c$ can be regarded as a continuous map $X \rightarrow \Pi G\left(F^{n}\right)$. Note that the metric on $G\left(F^{n}\right)$ (induced by the norm on $M_{n} F$ ) gives a metric on $\Pi G\left(F^{n}\right)$.

Since $\Pi G\left(F^{n}\right)$ is a metric space, it is paracompact. Let $S^{\prime}$ be a locally finite partition of 1 on $\Pi G\left(F^{n}\right)$ such that $|p-q|<1 / 9$ whenever $p$ and $q$ are in the same part. The map $c$ gives a locally finite partition $S$ of $X$ such that $|c(x, t)-c(y, t)|<$ $1 / 9$ for all $t$ whenever $x$ and $y$ are in the same part, i.e. $f(x) f(y) \neq 0$ for some $f \in S$.

For each $f$ in $S$ we pick a point $x=x(f)$ in $U(f)=\{z \in X: f(z) \neq 0\}$ and a positive number $\varepsilon(f)$ such that $|c(x, t)-c(x, s)|<1 / 9$ whenever $|t-s|<\varepsilon(t)$. Then

$$
\begin{aligned}
|c(z, t)-c(z, s)| & \leq|c(x, t)-c(x, s)|+|c(z, t)-c(x, t)|+|c(z, s)-c(x, s)| \\
& <1 / 9+1 / 9+1 / 9=1 / 3
\end{aligned}
$$

for every $z$ in $U(f)$ whenever $|t-s|<\varepsilon(f)$.

We set $\delta(z)=\sum_{f \in S} \varepsilon(f) f(z)$ for every $z$ in $X$. Then $\delta$ is a continuous positive function on $X$ such that $|c(z, t)-c(z, s)|<1 / 3$ whenever $|t-s|<\delta(z)$.

For every $z$ in $X$ and any integer $k \geq 0$ we set

$$
t_{k}(z)=\min (1, k \delta(z)), \quad c_{k}(z)=c\left(z, t_{k}(z)\right) .
$$

Then $c_{0}=a, c_{k}(z)=b(z)$ for $k \geq 1 / \delta(z)$, and $\left|c_{k}(z)-c_{k+1}(z)\right|<1 / 3$ for all $z$ and $k$.

We set

$$
g_{k}=c_{k} c_{k+1}+\left(1-c_{k}\right)\left(1-c_{k+1}\right) \text { for all } k .
$$

Then (see Lemma 4 and its proof) $c_{k} g_{k}=g_{k} c_{k+1},\left|1-g_{k}\right|<1$; hence $g_{k} \in \mathrm{GL}_{n}\left(F^{X}\right)$ for all $k$. Moreover, $g_{k}(z)=1$ for $k \geq 1 / \delta(z)$.

Finally, we set $G(z)=g_{0}(z) g_{1}(z) \cdots$. Then $g \in \mathrm{GL}_{n} F^{X}$ and $a g=g b$; hence $g^{-1} a g=b$. Lemma 8 is proved.

Theorem 2 is proved.

REMARK. Using that $G\left(F^{n}\right)$ is homotopically equivalent to $\left\{h=h^{*} \in \mathrm{GL}_{n} F^{X}\right\}$ we obtain that any two homotopic nonsingular hermitian forms over $F^{X}$ are isomorphic (when $X$ is compact, this is well known; see $[\mathbf{4}]$ ). In [1] the converse is claimed (that every two isomorphic forms are homotopic) when $F=C$ and $X$ is a finite $\mathrm{CW}$ complex. However, this claim is false, as the following counterexample shows. Let

$$
\begin{gathered}
X=S^{3}=\left\{\left(x_{i}\right) \in R^{4}: \sum x_{k}^{2}=1\right\}, \quad a=\left(\begin{array}{cc}
1 & 0 \\
0 & -1
\end{array}\right), \\
g=\left(\begin{array}{ll}
x_{1}+x_{2} i & x_{4} i-x_{3} \\
x_{3}+x_{4} i & x_{1}-x_{2} i
\end{array}\right) \in \mathrm{GL}_{2} F, \quad b=g^{*} a g .
\end{gathered}
$$

If $a$ and $b$ were homotopic, we would have $b=h^{*} a h$ with $h$ being the product of matrices close to 1 ; hence $h \in \mathrm{GL}_{1} F^{X}\left(E_{2} F^{X}\right)$. Then $g h^{-1} \in U\left(F^{X}\right) \subset$ 
$\mathrm{GL}_{1} F^{X}\left(E_{2} F^{X}\right)$; hence $g \in \mathrm{GL}_{1} F^{X}\left(E_{2} F^{X}\right)$. But homotopically $g$ is the identity map $S^{3} \rightarrow S^{3}$ and hence cannot be passed through the embedding $S^{1} \subset S^{3}$ (which corresponds to the embedding $\mathrm{GL}_{1} C \subset \mathrm{GL}_{2} C$ ). Of course, $a$ and $b$ are stably homotopic.

REMARK. Lemma 8 can be easily extended to an arbitrary Banach algebra $F$ (rather than $F=R, C$, or $H$ ). Namely, we can assert the following result.

THEOREM 9. Let $F$ be a Banach algebra with 1. Then for any topological space $X$ and any $n$, every two homotopic maps $X \rightarrow\left\{p=p^{2} \in M_{n} F\right\}$ give similar matrices in $M_{n} F^{X}$. Therefore there is a bijection between the finitely generated projective $F^{X}$-modules up to isomorphism and $\lim \pi\left(X,\left\{p=p^{2} \in M_{n} F\right\}\right)$. In particular, this set of isomorphism classes and, hence, $K_{0} F^{X}$ are homotopy-type invariants of $X$.

Moreover, checking the properties of $F$ which were actually used in the proof of Theorem 2, we see that we can replace the condition that $F$ is a Banach algebra with 1 in Theorem 9 by the following weaker condition: $F$ is a metrizable topological $R$-algebra with 1 such that the map $z \mapsto z^{-1}$ is defined and continuous in a neighbourhood of 1 .

4. Proof of Theorem 3. This theorem can be restated as follows. For any nonsingular hermitian form $\Phi$ on any object $P$ in $P\left(F^{X}\right)$ there is an orthogonal decomposition $P=P_{1} \oplus P_{2}$ such that the restriction of $\Phi$ on $P_{1}$ (resp. $P_{2}$ ) is positive (resp. negative); moreover, such a decomposition is unique up to isomorphism.

For compact $X$ this statement is Theorem 8.13 of [4]. This theorem is stated in [4] in terms of bundles, so the condition that $X$ is compact is needed to obtain a positive definite hermitian form. This condition can be replaced by the condition that the bundle is of finite type. Therefore to prove our Theorem 3 we can just repeat the arguments of $[\mathbf{4}]$.

REMARK. The referee attracted my attention to the fact that some ideas and computations in this paper can be traced back to the book Rings of operators by I. Kaplansky (Benjamin, 1968). In particular, our Lemma 4 is contained in Theorem 26 of the book. Also the referee pointed out the paper Partitions of unity in the theory of fibrations by A. Dold (Ann. of Math. 78 (1963), 223-255) which is one of the first studies of fibrations using locally finite partitions of unity.

\section{REFERENCES}

1. I. M. Gel'fand and A.S. Mishchenko, Quadratic forms over commutative group rings and the K-theory, Functional Anal. Appl. 3 (1969), 28-33. MR 41; Zbl. 250.55005

2. K. R. Goodearl, Cancellation of low-rank vector bundles, Pacific J. Math. 113 (1984), 289-302.

3. D. Husemoller, Fibre bundles, McGraw-Hill, New York, 1966.

4. M. Karoubi, K-theory, Springer, 1978.

5. R. G. Swan, Vector bundles and projective modules, Trans. Amer. Math. Soc. 105 (1962), 264-277.

6. __ Topological examples of projective modules, Trans. Amer. Math. Soc. 230 (1977), 201-234.

School of Mathematics, The Institute for Advanced Study, Princeton, NEW JERSEY 08540

Department of Mathematics, The Pennsylvania State University, UniverSITY PARK, PENNSYLVANIA 16802 (Current address) 